\title{
New techniques for auroral irregularity studies with COSCAT
}

\author{
P. Eglitis ${ }^{1 *}$, I. W. McCrea ${ }^{2}$, T. R. Robinson ${ }^{1}$, T. Nygrén $^{3}$, K. Schlegel ${ }^{4}$, T. Turunen ${ }^{5}$, T. B. Jones ${ }^{1}$ \\ ${ }^{1}$ Department of Physics and Astronomy, Leicester University, Leicester, LE1 7RH, UK \\ ${ }^{2}$ Rutherford Appleton Laboratory, Chilton, Didcot, UK \\ ${ }^{3}$ Physics Department, University of Oulu, Oulu, Finland \\ ${ }^{4}$ Max-Planck-Institut für Aeronomie, D-3411, Katlenburg-Lindau, Germany \\ ${ }^{5}$ Sodankylä Geophysical Observatory, Finland
}

Received: 27 October 1997 / Revised: 27 April 1998 / Accepted: 30 April 1998

\begin{abstract}
The COSCAT system enables the detection of E-region auroral backscatter with the EISCAT remote receivers at magnetic aspect angles close to $90^{\circ}$. This is achieved by utilising a low-power transmitter stationed in Oulu, Finland. Many important observations of E-region irregularities have been achieved with this simple experiment. Recent studies have attempted to push the COSCAT system to its experimental limits. Firstly, the CW signal has been phase-modulated with 13-bit Barker codes with baud lengths of 40, 70 and $100 \mu$ s. Interpretation of the received power allows the spatial distribution of the auroral scatterers to be determined. The second advance is in the use of a sophisticated correlator program which allows data to be buffered within the correlator at very high time resolution. This enables the coherent backscatter power to be sampled every $12.5 \mathrm{~ms}$ and the full auto-correlation function to be measured every $100 \mathrm{~ms}$. These measurements allow the COSCAT system to be employed for the first time in an investigation of the growth and decay of the auroral irregularities.
\end{abstract}

Key words. Ionosphere (ionospheric irregularities) Radio science (Instruments and techniques)

\section{Introduction}

The COherent SCATter experiment (COSCAT, McCrea et al., 1991) was developed to enable the observation of Eregion auroral plasma irregularities at small magnetic aspect angles with the European Incoherent SCATter facility (EISCAT; Rishbeth and Williams, 1985). It

\footnotetext{
* Present address: The Department of Astronomy, Uppsala University, Uppsala, Sweden

Correspondence to: P. Eglitis Swedish Institute of Space Physics, Uppsala Division, S-755 91 Uppsala, Sweden

e-mail: pxe@ion.le.ac.uk
}

comprises a low-power transmitter located in Oulu, Finland $\left(64.97^{\circ} \mathrm{N}, 25.67^{\circ} \mathrm{E}\right.$, see Fig. 1). The transmitter operates at a frequency of $929.5 \mathrm{MHz}$ and is connected to a dipole antenna array that forms a beam pointing at low elevations to the north. The illuminated volume is located on E-region altitudes between $72^{\circ}$ and $74^{\circ}$ latitude and $23^{\circ}$ and $27^{\circ}$ longitude. The EISCAT UHF receivers at Sodankylä, Finland, $\left(67.37^{\circ} \mathrm{N}, 26.65^{\circ} \mathrm{E}\right)$ and Kiruna, Sweden, $\left(67.86^{\circ} \mathrm{N}, 20.44^{\circ} \mathrm{E}\right)$ are oriented to look into the same volume. Under appropriate conditions auroral plasma irregularities, most recently reviewed by Haldoupis (1989) and Sahr and Fejer (1996), are generated. The irregularities are aligned with the geomagentic field and scatter the transmitted signal from Oulu towards the EISCAT remote receivers. The first observations comprised a passive use of the EISCAT system. These experiments determined the optimum observing geometry for the detection of coherent scatter, characterised the irregularity types (McCrea et al., 1991), and allowed a comparison with irregularities measured at other wavelengths to be made (Eglitis et al., 1995). Later developments included active experiments which studied the relationship of the irregularity characteristics to properties of the ionospheric plasma flow measured by incoherent scatter, (Eglitis et al., 1996).

Recent operation of the COSCAT experiment has focussed on improving the technical capabilities of the experiment, thereby furthering the study of auroral plasma irregularities. There are two key areas in which this has been achieved; Barker coding of the transmitted signal to allow the determination of spatial structure in the scattering region and the operation of special correlator programs at the EISCAT receivers in order to improve the time resolution of the observations.

\section{Description of the experiment}

COSCAT is an enhancement to the EISCAT facility that allows the study of auroral E-region plasma irregularities at UHF radar wavelengths. The plasma irregularities, which can be detected with COSCAT, have a 
nominal wavelength of $16 \mathrm{~cm}$. The two remote EISCAT receivers at Sodankylä and Kiruna are oriented to look at low elevations approximately due north (Fig. 1), into a volume of the E-region illuminated by a transmitting antenna at Oulu. The system has been fully described by McCrea et al. (1991) and the hardware specification is described by Thornhill and Chapman (1989). In this configuration the EISCAT remote receivers look almost perpendicular to the magnetic field where the scatter cross-section of the plasma irregularities is greatest. The Troms $\varnothing$ component of EISCAT is unable to look into this volume due to obscuration from local relief.

The scattering geometry has been discussed in detail by McCrea et al. (1991). In summary, the scattering volume for a given EISCAT remote receiver is determined by the volume intersection of the Oulu transmitter beam, the beam of the EISCAT receiver and the positions where the radar wave vector is close to orthogonal to the magnetic field direction. This effective scattering volume is approximately $800-1000 \mathrm{~km}$ north of the COSCAT transmitter in Oulu, between $72^{\circ}$ and $74^{\circ}$ geographic latitude. The volume is also constrained by the presence of irregularities. The Oulu transmitter beam has a $4^{\circ} 3 \mathrm{~dB}$ point separation. The EISCAT receiver beam width is much narrower, having a $0.6^{\circ}$ $3 \mathrm{~dB}$ point separation. If the irregularities are assumed to occupy a scattering layer $10 \mathrm{~km}$ thick, then the slant range extent of the EISCAT receiver beam that bisects the Oulu beam and contains irregularities is approximately $150 \mathrm{~km}$. This gives an upper limit on the slant range over which the COSCAT experiment can observe scatter. The geometric and aspect angle arguments imply

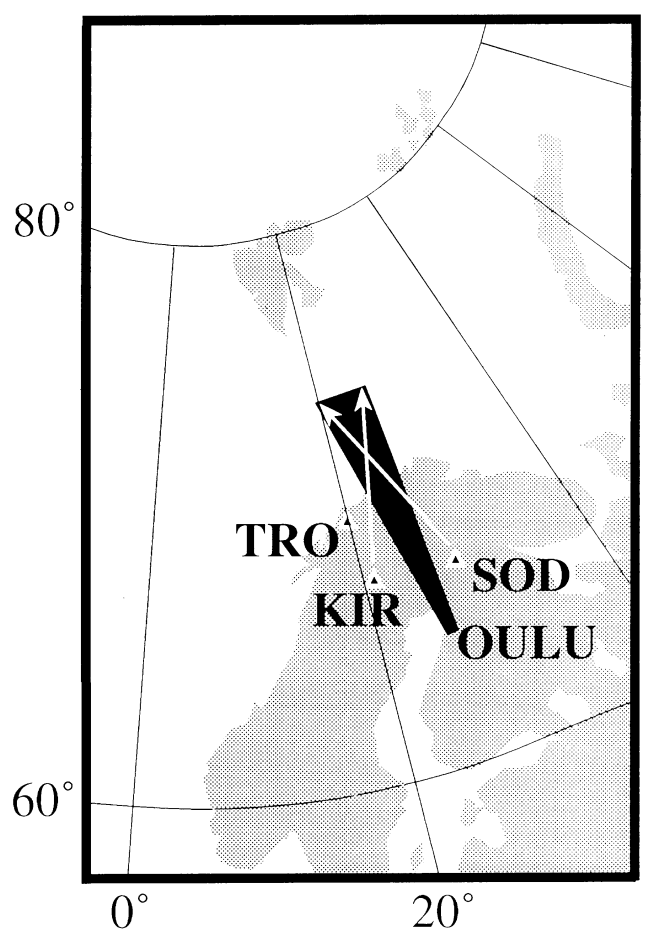

Fig. 1. A map illustrating the experimental geometry for the Barker and FAST-COSC experiments. The grid corresponds to geographic co-ordinates that this region is centred at a range of $900 \mathrm{~km}$ north of Oulu.

The two new techniques for obtaining improved spatial and temporal information are described below. The standard COSCAT experiment as previously employed by McCrea et al. (1991) is given the acronym COSC. The experiment employing Barker codes to discern spatial structure is labelled B-COSC and the high temporal resolution mode is called FAST-COSC. This terminology is intuitive and close to the original EISCAT experiment filenames: SP-UK-COSC, SP-UKBCOS and SP-UK-FASTCOSC.

\subsection{Spatial structure}

A limitation of the experiment is that there is no timing information available to relate a signal transmitted from Oulu to that detected at either of the EISCAT remote receivers. Hence, it was not possible to determine the absolute range of the scattering irregularities in previous experiments other than by the geometrical considerations already discussed.

The transmitter can only be operated in a continuous wave $(\mathrm{CW})$ mode. However, the transmitter design does allow the production of a CW 13-bit Barker coded phase modulation. The matched filters at the EISCAT receivers are capable of decoding this signal if it is detected. The detection of the Oulu signal is dependent on the presence of scattering targets in the EISCAT receiver and Oulu transmitter beam intersection. In this paper it will be demonstrated that information about the structure of the scattering region can be deduced from the correctly decoded signal. The Barker coding thus allows the determination of the spatial distribution and slant range extent of the scattering irregularities. This discrimination would be impossible with unmodulated CW transmission which has no absolute timing information.

The Barker code can be generated at a baud length between 20 and $100 \mu$ s (in steps of $10 \mu$ s). In experiments so far undertaken, baud lengths of 40,70 and $100 \mu$ s have been employed. These baud lengths are listed in Table 1 together with the equivalent spatial length of the code.

Barker coded experiments have been conducted during two intervals in May 1994 and January 1995. Two different programs were adopted at the EISCAT remote sites:

A: No attempt was made to decode the phase modulation of the received signal. The matched filter at the receiver was bypassed. The received signal was

Table 1. Barker baud periods and lengths

\begin{tabular}{lll}
\hline $\begin{array}{l}\text { baud period } \\
(\mu \mathrm{s})\end{array}$ & $\begin{array}{l}\text { baud length } \\
(\mathrm{km})\end{array}$ & $\begin{array}{l}\text { full code length } \\
(\mathrm{km})\end{array}$ \\
\hline 40 & 12 & 156 \\
70 & 21 & 273 \\
100 & 30 & 390 \\
\hline
\end{tabular}


sampled every $20 \mu \mathrm{s}$ and 64 lag auto-correlation functions (ACFs) were constructed. These were recorded at five second integration (COSC mode).

$\mathrm{B}$ : The signal was passed through the matched filter in order to decode the phase modulation. A 130-point data dump, containing ten repetitions of the 13-point Barker coded response function, was output every second (B-COSC mode).

Program B is an attempt to decode the signal received from a scattering target in the auroral ionosphere. Program A is the standard method for observing coherent scatter with the COSCAT system (e.g McCrea et al., 1991) and thereby detecting the presence of plasma irregularities. In some of the experiments, one of the EISCAT remote receivers would operate with program $\mathrm{A}$ in order to give an unambiguous indication of when plasma irregularities were present. Alternatively both receivers would operate program B. A summary of each Barker experiment is provided in Table 2. The operational modes of each receiver are stated for every experimental run. The numbers MMM in the string BCOSCMMM indicate the Barker code baud length adopted in $\mu$ s.

\subsection{High time resolution}

The temporal resolution of COSCAT measurements is limited to $1 \mathrm{~s}$ by the minimum dump time of the EISCAT receivers. However, Schlegel et al. (1990) adopted a special correlator program to make observations at sub-second resolution. The high temporal resolution is achieved by recording individual pulse returns in consecutive locations in the correlator memory and dumping the whole correlator memory every $5 \mathrm{~s}$. This program has been adapted for use with the

Table 2. Operations of the B-COSC experiment

\begin{tabular}{|c|c|c|c|}
\hline date (yy:mm:dd) & interval (UT) & Sodankylä & Kiruna \\
\hline $94: 05: 23$ & $1300-1600$ & B-COSC 100 & COSC \\
\hline $94: 05: 23$ & $2300-0500$ & B-COSC 100 & COSC \\
\hline $94: 05: 24$ & $1300-1600$ & B-COSC 100 & COSC \\
\hline $94: 05: 24$ & $2300-0500$ & B-COSC100 & COSC \\
\hline $94: 05: 25$ & $1300-1600$ & B-COSC 100 & COSC \\
\hline $94: 05: 25$ & $2300-0500$ & B-COSC 100 & B-COSC 100 \\
\hline 94:05:26 & $1300-1625$ & B-COSC 100 & COSC \\
\hline $94: 05: 26$ & $2025-0500$ & B-COSC 100 & COSC \\
\hline $94: 05: 27$ & $1300-1330$ & B-COSC 100 & COSC \\
\hline $94: 05: 27$ & $2300-0600$ & B-COSC 100 & COSC \\
\hline $94: 05: 28$ & $0500-0600$ & B-COSC40 & COSC \\
\hline $94: 05: 28$ & $1300-1725$ & B-COSC40 & COSC \\
\hline $94: 05: 28$ & $2300-0300$ & B-COSC40 & COSC \\
\hline $94: 05: 29$ & $0300-0500$ & B-COSC40 & B-COSC40 \\
\hline $94: 05: 29$ & $1300-1600$ & $\mathrm{COSC}$ & B-COSC40 \\
\hline $94: 05: 29$ & $2300-0200$ & $\mathrm{~B}-\mathrm{COSC} 40$ & $\mathrm{COSC}$ \\
\hline $94: 05: 30$ & $0200-0500$ & $\mathrm{~B}-\mathrm{COSC} 40$ & B-COSC40 \\
\hline $95: 01: 29$ & $0300-0500$ & $\mathrm{~B}-\mathrm{COSC} 70$ & $\mathrm{COSC}$ \\
\hline $95: 01: 29$ & $2300-0100$ & $\mathrm{~B}-\mathrm{COSC} 70$ & COSC \\
\hline $95: 01: 30$ & $0100-0300$ & $\mathrm{~B}-\mathrm{COSC} 70$ & B-COSC70 \\
\hline $95: 01: 30$ & $0300-0500$ & $\mathrm{~B}-\mathrm{COSC} 70$ & $\mathrm{COSC}$ \\
\hline $95: 02: 04$ & $2300-0500$ & B-COSC100 & COSC \\
\hline
\end{tabular}

COSCAT experiment, to make power measurements at $12.5-\mathrm{ms}$ resolution and measurements of the full ACF every $100 \mathrm{~ms}$. In addition, part of the correlator memory is still employed to record an ACF integrated to a 5-s resolution. The modified code was successfully run at EISCAT during a campaign of experiments between 7 and 14 March 1997. The experiment will be referred to as FAST-COSC. Observations were typically made in the interval 21:00 through to 05:00 UT on each night, since this was found to give the best occurrence statistics for backscatter. A similar strategy as adopted for the Barker experiment was applied to the FAST-COSC measurements. Typically, one remote receiver would run the FAST-COSC experiment while the COSC mode would be run at the other receiver, measuring backscatter spectra at 5-s temporal resolution. Only relative backscatter power values could be measured due to the lack of calibration of the separated transmitter and receiver system, unlike the measurements performed by Schlegel et al. (1990), which comprised absolute measurements of the backscatter cross-section with EISCAT. A summary of the experimental modes operated on the Sodankylä and Kiruna remote receivers is given in Table 3.

\section{Observations}

\subsection{Barker code results}

A typical set of observations is illustrated in Fig. 2. The top two panels depict output from the Sodankylä receiver from the B-COSC100 experiment undertaken on 24 May 1994. The top panel (Fig. 2a) contains a noisy series of powers, illustrating the data returned when there is no detection of plasma irregularities. During coherent scatter, a strong 13-bit periodicity is evident in the returned power (Fig. 2b). These two panels have been constructed by averaging the 13-bit response function of the Barker code on a given receiver channel for $1 \mathrm{~s}$ and plotting the resulting series of 13 samples for each second consecutively. This procedure creates a 'pseudo-time-series', in which each group of 13-points comprises data from a single integration period. The occurrence of coherent scatter was confirmed by running the COSC experiment at Kiruna. Parts $\mathrm{c}$ and $\mathrm{d}$ of Fig. 2 illustrate the Doppler spectra

Table 3. Operations of the FAST-COSC experiment

\begin{tabular}{llll}
\hline date (yy:mm:dd) & interval (UT) & Sodankylä & Kiruna \\
\hline 97:03:07 & $2100-2250$ & FAST-COSC & COSC \\
97:03:07 & $2250-0500$ & COSC & FAST-COSC \\
97:03:08 & $0000-0500$ & COSC & FAST-COSC \\
97:03:10 & $0000-0500$ & COSC & FAST-COSC \\
97:03:11 & $0300-0500$ & COSC & FAST-COSC \\
97:03:13 & $0000-0250$ & COSC & FAST-COSC \\
97:03:13 & $0250-0340$ & FAST-COSC & FAST-COSC \\
97:03:13 & $0340-0500$ & FAST-COSC & COSC \\
97:03:13 & $2100-0500$ & COSC & FAST-COSC \\
\hline
\end{tabular}



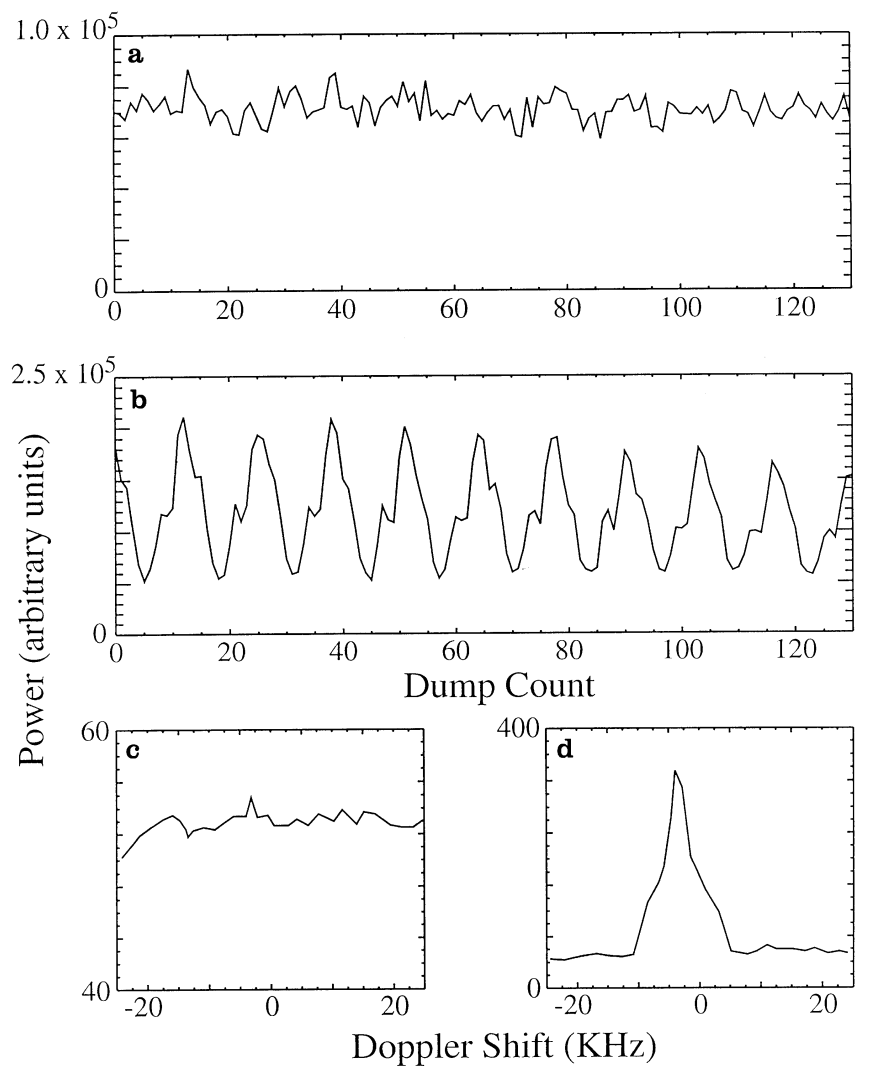

Fig. 2. a typical noisy returns for the Barker part of the experiment are depicted with the corresponding COSC spectrum in panel c. During the observation of coherent spectra at Kiruna, panel d, a 13-point periodicity is observed in the Barker decoded signal detected at Sodankylä, panel $\mathbf{b}$. In the $x$-axis label of panels a and b one 'Dump Count' is the 1-s average of one bit of the Barker code

observed at Kiruna during the same intervals as the data in a and b, respectively, of Fig. 2.

When coherent backscatter was detected during the $100-\mu$ s observations, the Barker decoded signal exhibited many different 13-point periodicities. These varied from almost purely sinusoidal forms to multiple-peaked shapes. At different baud lengths systematic differences in the observed 13-bit response function were discovered. In Fig. 3 results from observations at baud lengths of 40, 70 and $100 \mu$ s from campaigns run in 1994 and 1995 are presented. Each panel depicts a time-series of 5$\mathrm{s}$ averages of the 13-bit response function of the Barker code, sampled at Sodankylä. At $100 \mu$ s (bottom panel) the 13-bit pattern exhibits clear reproducible structure and a large dynamic range, i.e. the minimum power for each consecutive 13-points is at the noise level of the radar returns and this minimum is independent of the maximum power observed in each 13-point series. In contrast, at the shortest baud length ( $40 \mu$ s top panel) an increase in power is observed for all 13-points when coherent scatter first commences at around 03:36:45 UT. Structure is evident in the response function, but it is in no way as distinct as that for the longest baud length observations. For an intermediate baud length of $70 \mu \mathrm{s}$ the response function is essentially flat and discrete steps
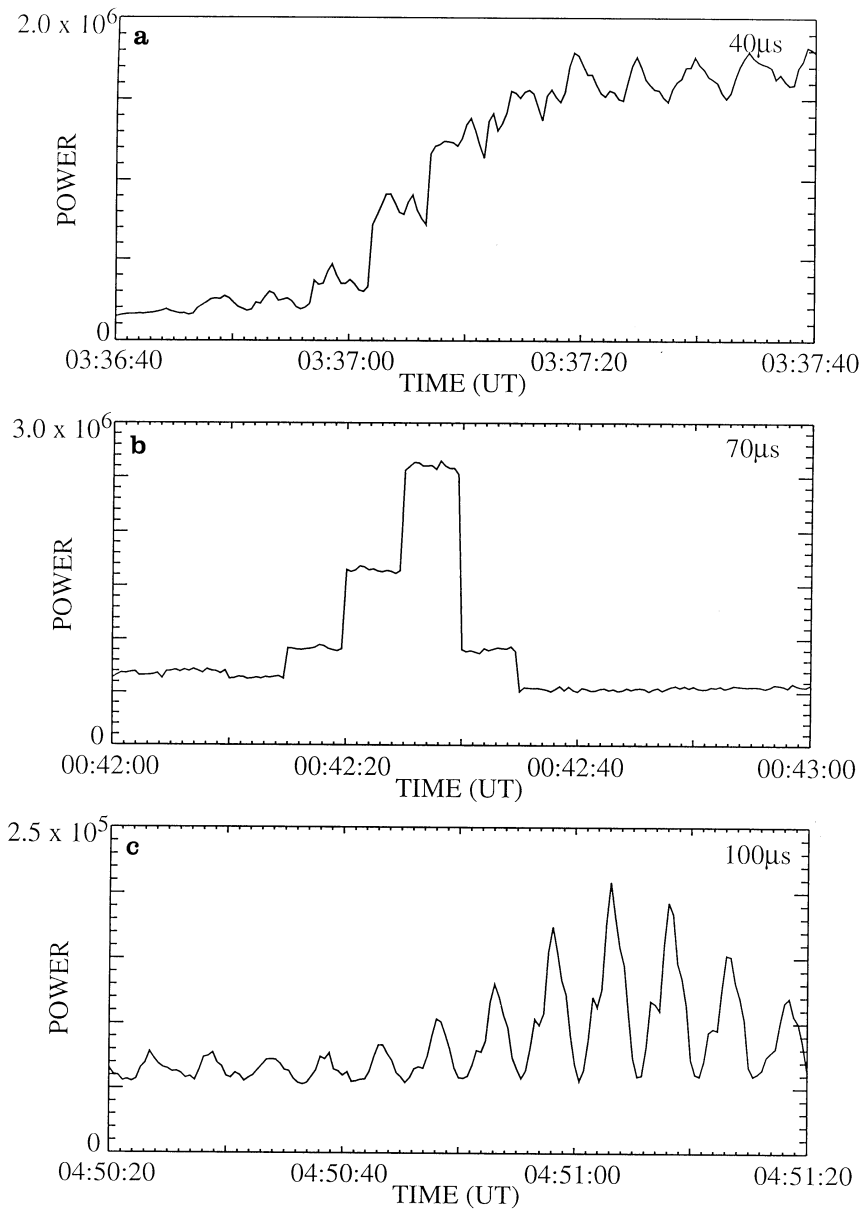

Fig. 3a-c. A comparison of observations at a 40, b 70 and c $100 \mu \mathrm{s}$ baud period

in power between successive 13-point samples are observed.

For each of the data sets presented in Fig. 3 the occurrence of coherent scatter was confirmed by COSC observations from Kiruna. The observed spectra and $\mathrm{ACF}$ also exhibit features pertaining to the transmission of the Barker code. Examples of the ACFs and spectra for observation at each baud length are presented in Fig. 4. The effect of the Barker code upon the ACF appears as a contamination at lags corresponding to multiples of the Barker code length. For each COSC experiment the ACFs were calculated from samples measured at a spacing of $20 \mu \mathrm{s}$. A 13-bit Barker code with $40-\mu$ s baud length has a total code length of $520 \mu \mathrm{s}$, which corresponds to the 26th lag of the ACF. The ACF in the top panel, Fig. 4a, was measured at Kiruna during a $40-\mu$ s experiment. There is clear contamination in lags 26,27 and 28 and also near lag 52. The contamination is also evident in the backscatter spectrum (Fig. 4b) as ringing. At long baud lengths $(100 \mu \mathrm{s})$ the ringing is not resolved and the contamination appears as a skirt on the spectrum, Fig. 4d. For the intermediate baud length, e.g. Fig. $4 \mathrm{c}, 70 \mu \mathrm{s}$, the contamination does not occur and a typical type-1, (Bowles et al., 1960) irregularity spectrum is measured. 

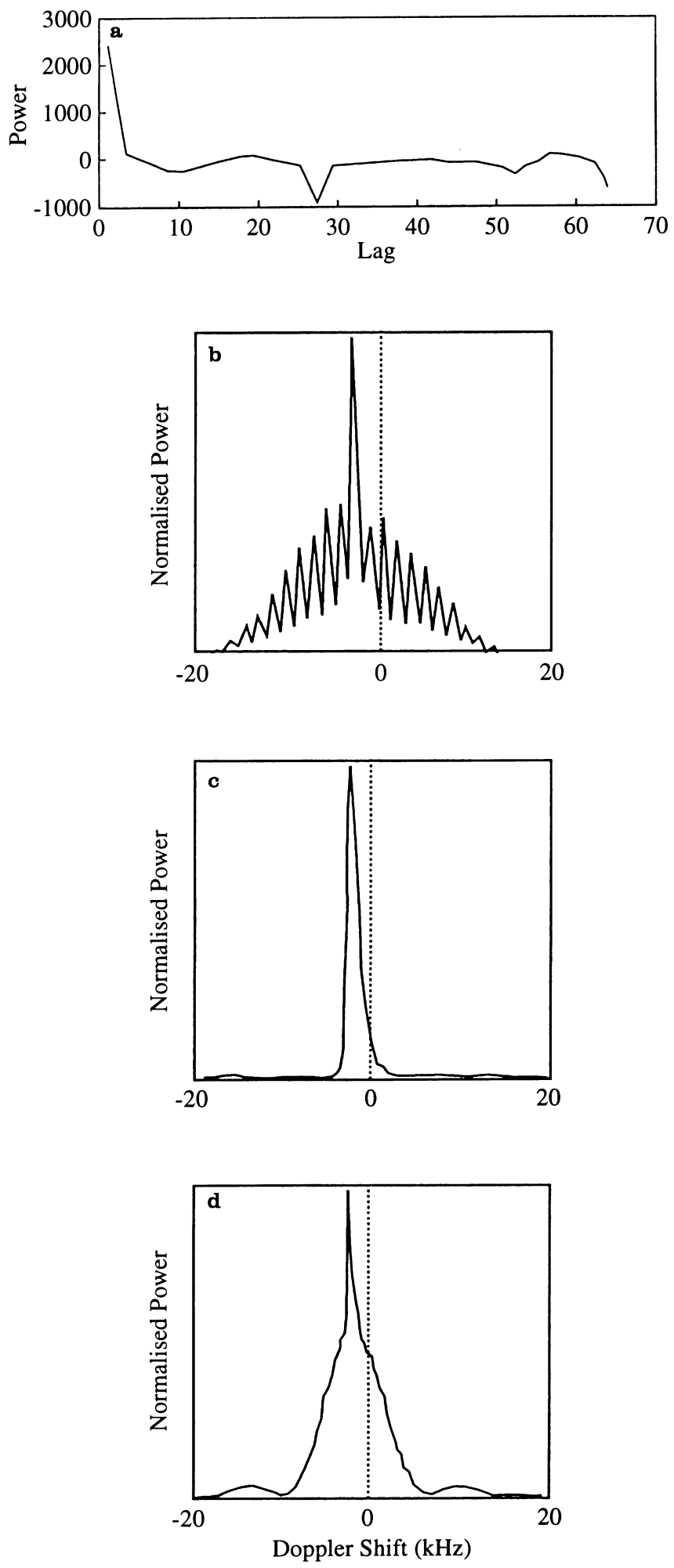

Fig. 4. a depicts an ACF observed by the COSC experiment when a 40- $\mu$ s Barker code was being transmitted. b, c and d, exhibit the measured spectrum at Kiruna for 40,70 and $100 \mu$ s, respectively

\subsection{FAST-COSC results}

Auroral scatter was first observed with the FAST-COSC experiment at 22:09 UT on 7 March, 1997. Time-series of this interval of data are depicted in Fig. 5. During this
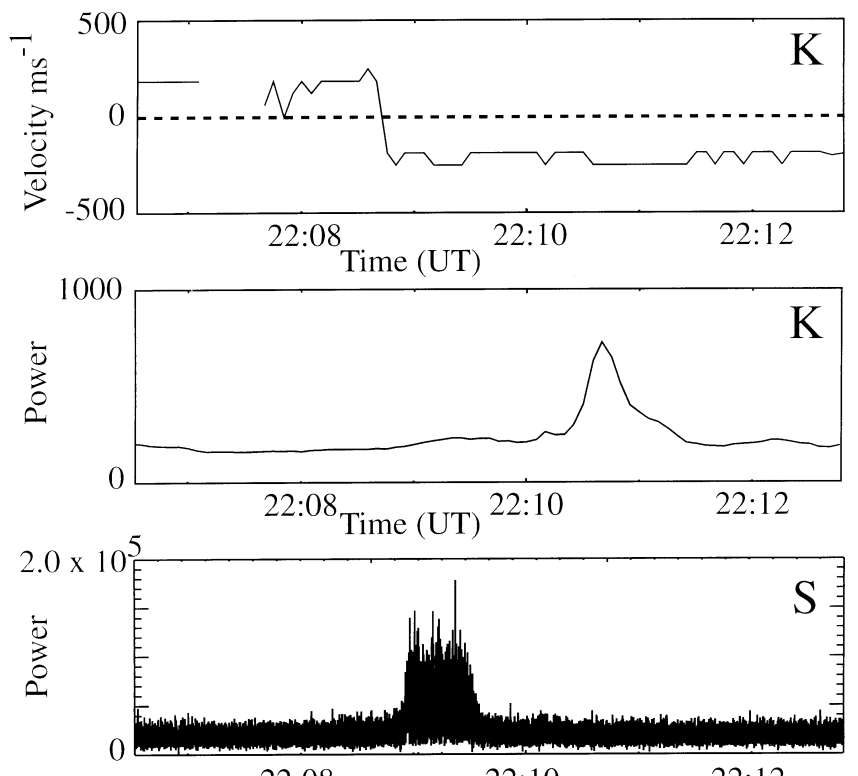

22:08 Time (UT) $22: 10$
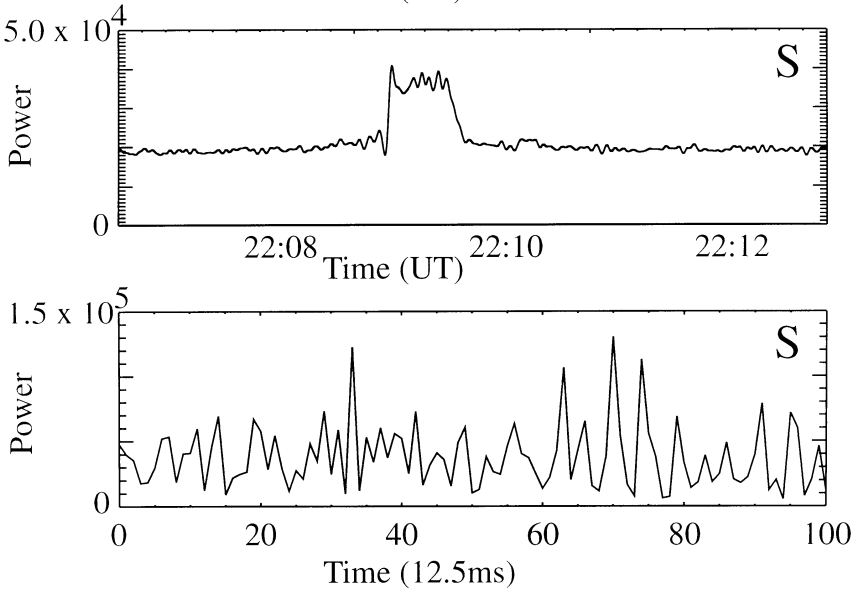

Fig. 5. FAST-COSC observations for 7 March 1997. From top to bottom the panels illustrate: the COSC determined irregularity phase speed measured at the Kiruna receiver, the COSC measured backscattered power, the $12.5-\mathrm{ms}$ resolution slicer profile measured by the FAST-COSC experiment at Sodankylä, the same time-series filtered to remove high-frequency components and a 100-point sample of data from within the scattering event, commencing at approximately 22:09:20 UT

interval, the Kiruna receiver was running the 5-s resolution COSC mode and the Sodankylä receiver performed the FAST-COSC experiment.

The top two panels, of Fig. 5, labelled K, depict the Kiruna radar observations of irregularity phase speed and backscatter power. The Kiruna receiver observes backscatter continuously from 22:08 UT. Initially the phase speed is approximately $200 \mathrm{~m} \mathrm{~s}^{-1}$. Just before 22:09 UT, an apparent reversal is seen in the direction of the phase velocity, whereafter the phase speed is between -200 and $-250 \mathrm{~m} \mathrm{~s}^{-1}$. At 22:10:30 UT a sudden increase in power occurs followed, at first by a fast decrease and then a much more gradual decrease. The duration of this increase in power comprises a significant scattering event. 
The third panel of Fig. 5 illustrates the high-timeresolution power time-series measured at the Sodankylä receiver. Here a similar scattering event is observed. There is a sudden increase in power at just before 22:09 UT. The power remains increased for $40 \mathrm{~s}$ until it falls, in about $10 \mathrm{~s}$, back to the noise level. The duration and time evolution of the events as observed by the Kiruna and Sodankylä receivers are the same, but the occurrence time is $60 \mathrm{~s}$ earlier at Sodankylä. The signal detected at Sodankylä is very noisy, exhibiting large fluctuations between high signal power and noise. In panel four the signal has been filtered to remove the high-frequency components better to illustrate the time evolution of the observed power. There does appear to be a sudden impulsive increase in power which remains sustained for a short period until it decreases back to the noise level. A 1.25-s sample of the FAST-COSC data is presented in the bottom panel. The data sample commences at approximately 22:09:20 UT. Here there may be evidence of individual scatterers (e.g. commencing at point 68 and point 73 on the $x$-axis) that grow in periods equal to or less than the sampling time $(12.5 \mathrm{~m} \mathrm{~s})$ and decay back to the noise in about $30 \mathrm{~ms}$, giving approximately $40-\mathrm{ms}$ lifetimes in all. This lifetime estimate is derived from just considering the large and sustained observations of power. There are large individual spikes in the time-series, which may indicate lifetimes of the order $20 \mathrm{~ms}$ and other intervals of slightly increased power giving estimates up to $60 \mathrm{~ms}$. A more detailed analysis of such data sets is the subject of future work.

The real parts of the ACFs observed at Sodankylä during this interval are illustrated in Fig. 6. Both 5-sintegration ACFs (top row) and 100-ms ACFs (second row) are illustrated. In the third and fourth rows of Fig. 6 the phase angle at each lag of the ACF is plotted for the 5-s and 100-ms observation, respectively. The gradient of the phase angle variation with lag is proportional to the phase velocity of the observed irregularity. The analysis of the ACF suggests that there is little difference between the ACFs at each time resolution; both exhibit similar decorrelation times and give the same phase velocity. The 100-ms ACFs are noisier than the 5-s ACFs as would be expected from the difference in integration time. However, it is interesting to note that individual $100-\mathrm{ms}$ ACFs are often as well defined as their 5-s-resolution counterparts, e.g. the middle example of a 100-ms ACF in Fig. 6.

The lag separation in the ACF is $20 \mu \mathrm{s}$, the ACFs decorrelate almost fully at the 50th lag, this gives an approximate decorrelation time of $50 \times 20 \mu \mathrm{s}=1 \mathrm{~ms}$. This is much smaller than the lifetime of the individual scattering events $(40 \mathrm{~ms})$ observed in the FAST-COSC time-series.

The scattering event presented in Fig. 5 is the only instant when backscatter was evident in the FASTCOSC data at the $12.5-\mathrm{ms}$ resolution. However, auroral scatter was observed over many more intervals by the COSC part of the experiment. Post-integration of FAST-COSC data for these times, also revealed the presence of auroral scatter. A sample of post-integrated data is presented in Fig. 7 taken from an experiment run during the morning of 13 March 1997. The top panel contains the backscatter power observed at Sodankylä where the COSC mode was operated. The bottom panel contains the post-integrated FAST-COSC data for the same interval, at a resolution of $5 \mathrm{~s}$. Comparing the top and bottom panel it is evident that both the Kiruna and Sodankylä receiver are detecting the same approximate variation in backscatter power, although the signal to noise ratio is smaller at Kiruna, and the fluctuations are observed at the same time at both receivers.

\section{Discussion}

\subsection{Barker experiment}

The 13-bit response function is the cross-correlation of the transmitted signal with the distribution of scattering targets in the radar beam. A simple algorithm was coded, with the Matlab programming language, to calculate this response for a given set of irregularity distributions and baud lengths.

An instructive set of simulation results is depicted in Fig. 8. The column of left-hand panels illustrates various modelled distributions of scattering irregularities with respect to slant range. Superposed on these is a sketch of a single transmitted code, the spatial coverage of which is drawn to the same scale. The right-hand panels give the simulated decoded signal that the scattering layer would produce at the EISCAT remote site, with ten repetitions of the Barker code response function. In the simulation the code baud length was kept fixed at $100 \mu \mathrm{s}$ and the slant range extent of the scattering irregularities was varied. This is equivalent to keeping the range extent of the scatterers constant and varying the baud length.

In Fig. 8a the code length is greater than the scattering region which comprises a Gaussian profile. The modelled received power exhibits the same distribution of power in each 13-point dump. If some structure is introduced to the Gaussian profile, e.g. Fig. 8b, the same structure arises in the Barker response function. Thus, when the code length is greater than the scattering region the response function is equivalent to the power profile of the scattering region. Increasing the profile extent to a value greater than the code length produces the result illustrated in Fig. 8c; the shape of the response function is more complicated and the dynamic range of the received signal is much smaller. If the code length is equal to twice the range extent of the scattering region, no structure is observed in the returned power (Fig. 8d).

The transmitted signal is a $\mathrm{CW}$ sequence of Barker codes. As the signal propagates, backscatter from points separated in slant range by half the spatial extent of the Barker code will reinforce. Let $L_{1 / 2}$ represent this distance. If the scatterers occupy a region of space less than $L_{1 / 2}$ in extent, only power from one repetition of the code will ever be returned. As the continuous sequence of codes moves through the scattering layer, 

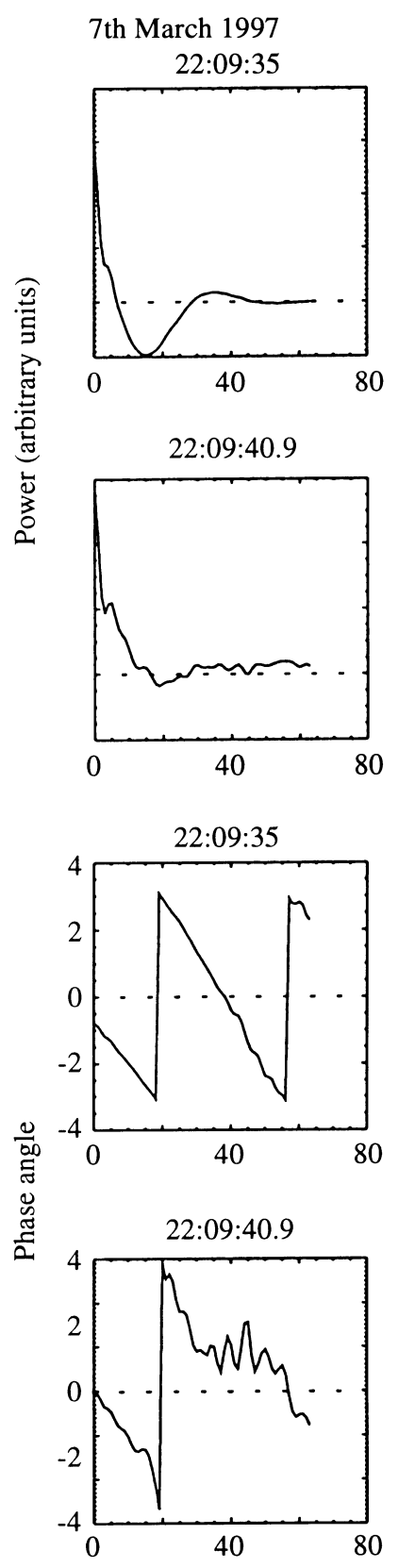
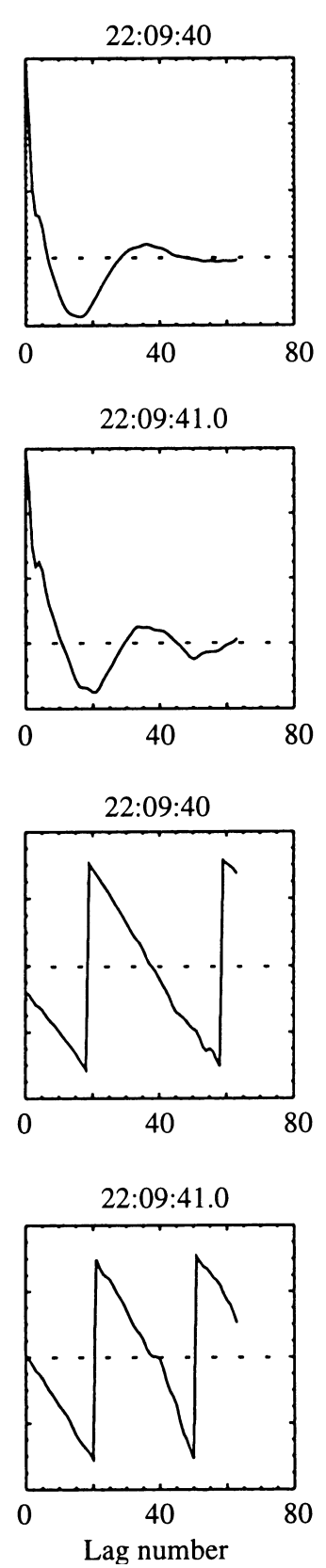
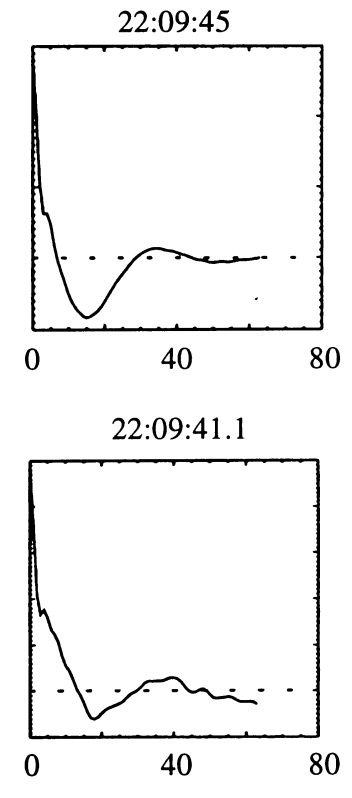

22:09:45
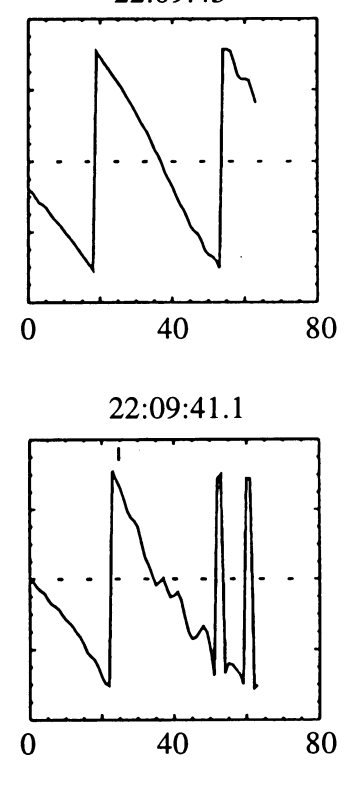

Fig. 6. A comparison of low (1st and 3rd row) and high (2nd and 4 th row) time resolution ACFs and the phase angles calculated from the ACF the spatial structure of the narrow scattering region will be repeatedly mapped out. The simulation results presented in Fig. 8a and b and the observation described in Fig. $3 \mathrm{c}$ are consistent with the condition $L_{1 / 2}>$ slant range extent of the scatterers.

If the slant range extent of the scattering region is equal to an integer multiple of $L_{1 / 2}$ then all the bauds from a complete Barker code are always scattered back to the receiver, and the decoded signal power from a uniform layer would be constant as illustrated in Fig 8d. If the irregularities occupy a slant range length greater than $L_{1 / 2}$ then more than one Barker code is scattered back simultaneously, e.g. Fig. 8c.

The results of the model explain the differing observations at each baud length as illustrated in Fig. 3. The curious observation at $70 \mu$ s (Fig. 3b) simply arises since the slant range extent of irregularities is approximately equal to (or a multiple of) $L_{1 / 2}$. The response is essentially flat for each 13-point dump (i.e. very small dynamic range). There are evidently changes in the mean irregularity cross-section between successive 13-point dumps, which accounts for the steps in power between each integration period. The baud lengths of 100 and 40 $\mu$ s give code lengths longer and shorter than the scattering region slant range extent, respectively.

As described in Sec. 2, the slant range extent of the EISCAT beam that bisects the Oulu beam and can contain irregularities is approximately $150 \mathrm{~km}$. This length is close to the $L_{1 / 2}$ condition for the $70-\mu$ s code. Therefore, it is the experimental geometry that forces the 

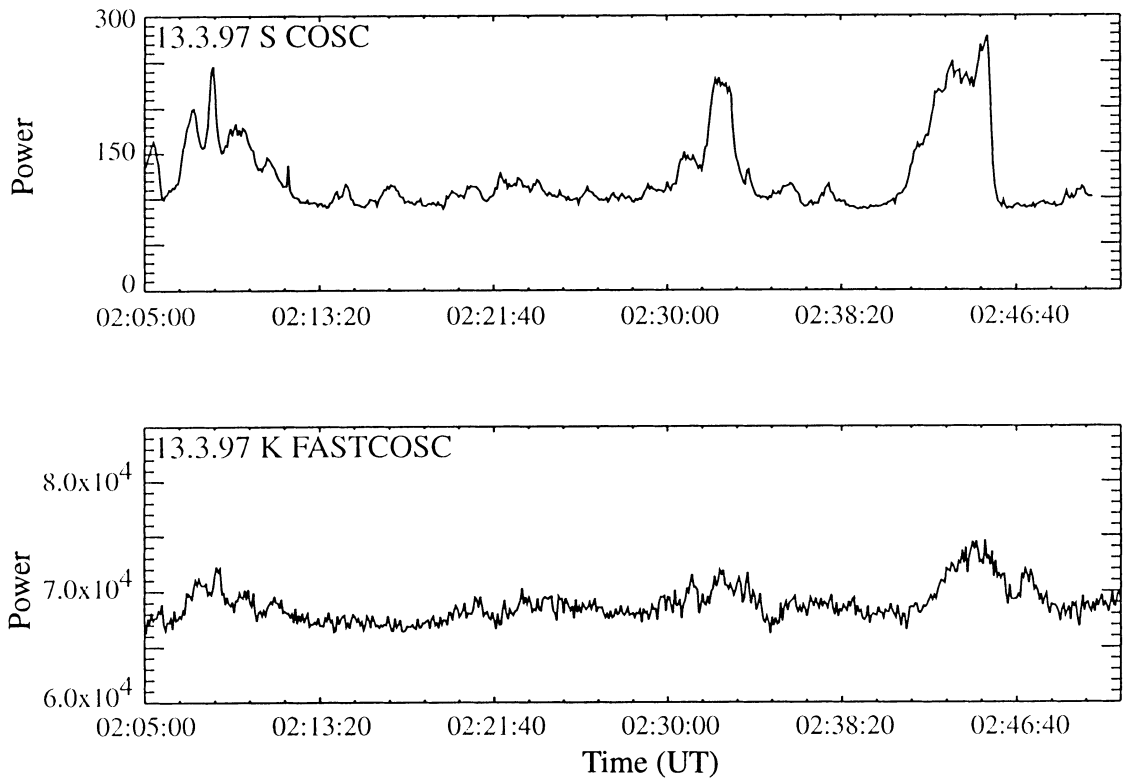

Fig. 7. COSC and FAST-COSC data from 13 March 1997. Top panel : COSC power; bottom panel : 5-s post integrated FASTCOSC observations
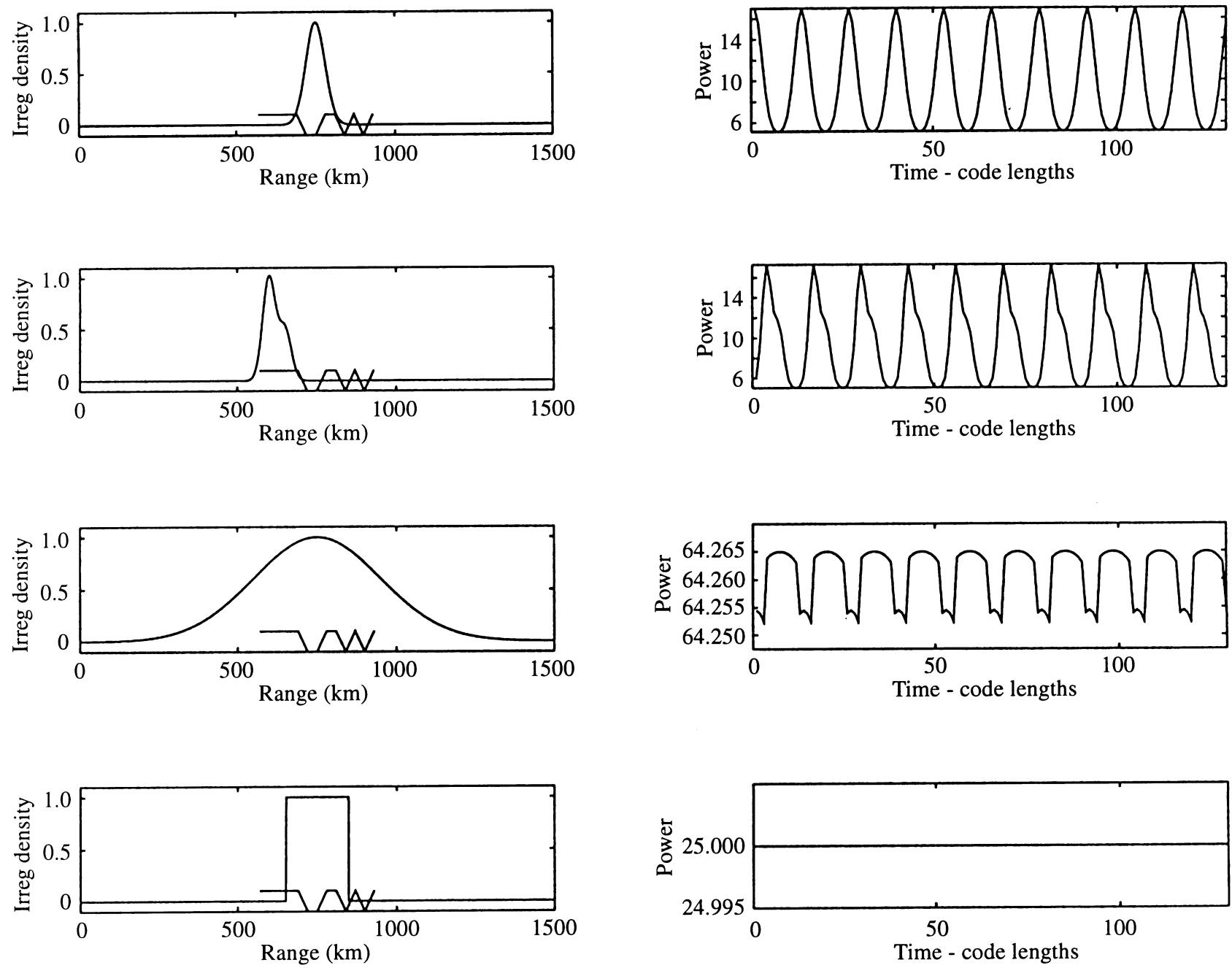

Fig. 8. A set of simulation results for differing distributions of irregularities with range. The left-hand column depicts a modelled range distribution of irregularities with a full Barker code sequence

superimposed to the correct scale. The right-hand column illustrates ten cycles of the modelled Barker response function. The reader should note the different $y$-axis scales in the right-hand column of panels 
matching of code length to irregularity range extent and this implies that the entire EISCAT beam is full of scattering irregularities.

An interesting example was observed in an experiment run on the 29 January 1995 at $70 \mu$ s depicted in the top panel of Fig. 9. Throughout the interval presented in the figure, coherent scatter was observed at the Kiruna receiver, where the COSC program was in operation. Examples of three observed spectra are pictured in the second panel. No 13-point periodicities were observed in the Sodankylä measurements except during decreases in received power. This indicates a contraction in the extent of the scattering region, such that it no longer satisfied the condition where the range extent was equal to a multiple of $L_{1 / 2}$. Under these conditions a decrease in received power would be detected along with the appearance of a 13-point periodicity. This is correlated with the spectral variations observed at Kiruna. At 04:40:10 UT and 04:48:20 UT when there was no periodic structure observed in the B-COSC power, the spectra at Kiruna are narrow and well defined. At 04:44:20 UT, when there is a minimum in the received B-COSC power, the Kiruna receiver also detects less backscatter power and the spectra are contaminated by the Barker code modulation. The contamination of the COSC spectra can be explained as follows. If all the bauds in the transmitted signal are received equally at any given time, then sampling the return signal (with no matched filter) will not detect the 13-bit Barker modulation. When the distribution of scattering targets does not match the code length, then the contributions from each baud to each sample will vary with time and the 13-bit periodicity will be identified.

\subsection{FAST-COSC experiment}

The FAST-COSC results share many similarities with previous high-resolution and $\mathrm{CW}$ observations. The
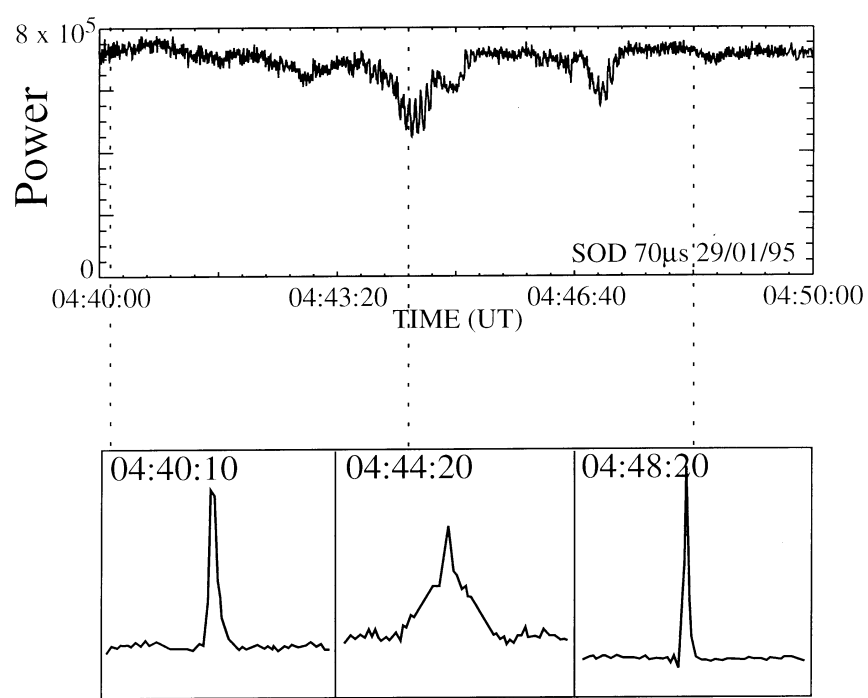

Fig. 9. An interesting example of data where the returned power reveals structure and periodicity during decreases in the received power. At selected times, the corresponding COSC spectrum is also plotted observed ACFs at $5 \mathrm{~s}$ and $100 \mathrm{~ms}$ have similar shapes in agreement with the observations of Schlegel et al. (1990), who employed EISCAT alone to detect coherent scatter and were limited to observations between $4^{\circ}$ and $6^{\circ}$ from magnetic orthogonality although they had a 2MW transmitter at their disposal. This is in contrast to the FAST-COSC observations near magnetic orthogonality but with only a $300-\mathrm{W}$ transmitter power.

Analysis of the FAST-COSC 12.5 -ms time-series suggests there are individual scatterers with lifetimes of the order of 20-60 ms. Hall et al. (1993) conducted a detailed set of $\mathrm{CW}$ experiments at $50 \mathrm{MHz}$, explicitly measuring the lifetimes of auroral scatterers. They found evidence for the presence of strong single scatterers with mean lifetimes of the order of $50 \mathrm{~ms}$. In addition, highresolution data from the $50-\mathrm{MHz}$ SESCAT radar (Haldoupis and Schlegel 1993), exhibit lifetimes below $100 \mathrm{~ms}$ for E-region irregularities observed at midlatitudes (Schlegel, private communication). Although the FAST-COSC results are based upon only a few observations, the preliminary results suggest that the lifetime of the irregularities at $16-\mathrm{cm}$ wavelength is of the same order as the Hall et al. (1993) results for a wavelength of $3 \mathrm{~m}$. The typical decorrelation time of a COSCAT ACF is about $1 \mathrm{~ms}$. This is much smaller than any of the estimated lifetimes from the FAST-COSC time-series. This discrepancy between time-scales implies that relative to the correlation time of velocity fluctuations in the ionospheric plasma, the irregularities comprise stable scattering targets. The ACF decorrelates due to the turbulent plasma flow and not the lifetime of the irregularity. We present this as the most likely reason for the difference between the ACF decay time and the FAST-COSC lifetimes. However, other causes for the decorrelation, such as interference between different scatterers, cannot be discounted.

The simultaneous use of the FAST-COSC mode on one of the EISCAT remote receivers and the COSC mode on the other enables us to study the backscattering irregularities in two different ways. The volume illuminated by the Oulu transmitter is much greater than the beamwidths of the EISCAT remote receivers, which only intersect in a small portion of the illuminated volume, as indicated by Fig. 1. Thus it would seem logical that a small and isolated auroral target might not be seen simultaneously at the two remote sites, as such a feature is statistically unlikely to pass through the intersection region. However, backscatter would be seen simultaneously at the two sites if the scatterers, instead of being a small discrete target, filled a large part of the illuminated volume.

Both of the cases described above can be seen in the data presented here. In Fig. 7, the occurrence of coherent scatter correlates well between the remote sites and there is fair agreement between the observed temporal behaviours, suggesting that a large part of the illuminated region is filled with irregularities. However, the result illustrated by Fig. 5 is consistent with a small region of strong scatter moving through the illuminated region from east to west, first passing through the Sodankylä beam and then through the 
Kiruna beam. The sense of this motion is consistent with the expected background flow at the time of the event. Although the backscatter power envelope observed at each radar is very similar, suggesting a stable drifting structure, the detailed 12.5 -ms data reveal the scattering process to comprise bursts of individual scattering events and complex temporal structure.

\section{Conclusion}

Two novel solutions to the hardware limitations of the COSCAT system have been developed and successfully run. Detailed information about the spatial distribution and time evolution of 16-cm-wavelength auroral plasma irregularities can now be gleaned and this paper has presented initial results and studies using these new data sets. Future work will present more detailed investigations of the collected observations, important studies include a comparison of the inferred plasma flow velocity with the spatial structure derived from the Barker observations and a cross-correlation of the observations at each EISCAT receiver using the FASTCOSC and Barker data. A compelling future experiment would be to make both Barker and FAST-COSC observations simultaneously.

It has been established that the use of long Barker codes, e.g. 100- $\mu$ s baud length, allow the determination of a power profile through the scattering region with respect to slant range. In addition, it is known that the scattering region is centred at $900 \mathrm{~km}$ North of Oulu and is of the order of $200 \mathrm{~km}$ in length. If a 13-point power profile is measured in this volume, then a range resolution of approximately $15 \mathrm{~km}$ can be determined.

An estimate of the 16-cm-wavelength irregularity lifetime is of the order $40 \mathrm{~ms}$. This is very similar to the lifetime of the 3-m-wavelength irregularities observed by Hall et al. (1993). However, it should be emphasised that the purpose of this initial paper is to present the new techniques and preliminary results. The $40-\mathrm{ms}$ lifetime is a tentative result, a more detailed statistical analysis of the FAST-COSC time series will be conducted in future work. The observed irregularity spectral shape is very similar to the results obtained by Schlegel et al. (1990), whose observations were made at the same wavelength, but $5^{\circ}$ further from magnetic orthogonality.

The Barker and FAST-COSC observation both indicate that often the illuminated volume is completely full of scattering irregularities although at other times localised targets drift through the volume. The high temporal resolution data suggests that for both of these conditions the backscatter is dominated by individual scatterers that grow and decay from the noise, e.g. bottom panels of Figs. 5 and 7. The 100-ms ACFs also show variability from sample to sample, alternating between noisy and more well-defined ACFs. At longer integrations this variability is naturally averaged out.
However, the underlying question is whether the radar field of view is dominated by a small number of strong targets, growing and decaying continuously, or a large number of smaller cross-section plasma waves. It is very difficult to separate these two regimes, but this work and that of Hall et al. (1993) suggest the former case may be more likely.

Acknowledgements. The authors thank the Director of EISCAT and all the EISCAT staff for the operation of EISCAT and supplying the data. The EISCAT association is supported by the national scientific agencies of Finland, France, Germany, Norway, Sweden, Japan and the United Kingdom. The Rutherford Appleton Laboratory EISCAT group are thanked for handling the data and collaboration in organising the experimental campaigns. The authors also express their gratitude to Mr. Pabst, MPAE for the COSCAT antenna construction and E. C. Thomas, J. D. Thornhill and P. J. Chapman, University of Leicester, for the design and construction of the transmitter. Professor J. Oksman, University of Oulu, and his staff are thanked for providing a site for the transmitter and help in running experiments.

Topical Editor D. Alcaydé thanks G. Sofko and J.D. Sahr for their help in evaluating this paper.

\section{References}

Bowles, K. L., R. Cohen, G. R. Ochs, and B. B. Balsley, Radar echoes from field-aligned ionization above the magnetic equator and their resemblance to auroral echoes, J. Geophys. Res., 65 , 1853-1855, 1960.

Eglitis, P., T. R. Robinson, I. W. McCrea, K. Schlegel, T. Nygrén, and A. S. Rodger, Doppler spectrum statistics obtained from 3 different-frequency radar auroral experiments, Ann. Geophysicae, 13, 56-65, 1995.

Eglitis, P., I. W. McCrea, T. R. Robinson, K. Schlegel, T. Nygrén, and T. B. Jones, Flow dependence of COSCAT spectral characteristics, J. Atmos. Terr. Phys., 58, 189-203, 1996.

Haldoupis, C., A review on radio studies of auroral E-region ionospheric irregularities, Ann. Geophysicae, 7, 239-258, 1989.

Haldoupis, C., and K. Schlegel, A 50-MHz radio Doppler experiment for mid-latitude E-region coherent backscatter studies: system description and first results, Radio Sci., 28, 959-9788, 1993.

Hall, G. E., D. Andre, D. W. Danskin, G. F. Sofko, and J. A. Koehler, Lifetime measurements of auroral scatterers, J. Geophys. Res., 98, 17 537-17 542, 1993.

McCrea, I. W., K. Schlegel, T. Nygrén, and T. B. Jones, COSCAT, a new auroral radar facility on $930 \mathrm{MHz}$ - system description and first results, Ann. Geophysicae, 9, 461-469, 1991.

Rishbeth, H. and P. J. S. Williams, The EISCAT ionospheric radar: the system and its early results, $Q$. J. R. Astron. Soc., 26, 478$512,1985$.

Sahr, J. D. and B. G. Fejer, Auroral electrojet plasma irregularity theory and experiment: a critical review of present understanding and future directions, J. Geophys. Res., 101, 26 893-26 909, 1996.

Schlegel, K., T. Turunen, and D. R. Moorcroft, Auroral radar measurements at $16-\mathrm{cm}$ wavelength with high range and time resolution, J. Geophys. Res., 95, 19 001-19 009, 1990.

Thornhill, J. D., and P. J. Chapman, COSCAT operating and service manual, Ionospheric Physics Group Technical Report, University of Leicester, UK, September 1989. 